\title{
siRNA against the $G$ gene of human metapneumovirus
}

Faith Maxine Preston ${ }^{1,2}$, Claire P Straub ${ }^{1,2}$, Ruben Ramirez ${ }^{3}$, Suresh Mahalingam ${ }^{4}$ and Kirsten M Spann ${ }^{1,2^{*}}$

\begin{abstract}
Background: Human metapneumovirus (hMPV) is a significant viral respiratory pathogen of infants and children, the elderly and immunocompromised individuals. Disease associated with hMPV infection resembles that of human respiratory syncytial virus (RSV) and includes bronchiolitis and pneumonia. The glycosylated G attachment protein of hMPV is required for viral entry in vivo and has also been identified as an inhibitor of innate immune responses.
\end{abstract}

Findings: We designed and validated two siRNA molecules against the G gene using A549 cells and demonstrated consistent 88-92\% knock-down for one siRNA molecule, which was used in subsequent experiments. Significant reduction of G mRNA in A549 cells infected with hMPV did not result in a reduction in viral growth, nor did it significantly increase the production of type I interferon $(\alpha / \beta)$ in response to infection. However, there was a moderate increase in IFN- $\beta$ mRNA expression in response to infection in siG-transfected cells compared to untransfected and si-mismatch-transfected cells. Expression of $\mathrm{G}$ by recombinant adenovirus did not affect type I IFN expression.

Conclusion: $\mathrm{G}$ has been previously described as a type I interferon antagonist, although our findings suggest it may not be a significant antagonist.

Keywords: siRNA, hMPV, Type I interferon

\section{Findings}

\section{Background}

Human metapneumovirus (hMPV) is a member of the Pneumoviridae subfamily of the Paramyxoviridae [1]. hMPV infects half of all infants under the age of 1 year and almost all children have been infected by the age of 10 years [2,3]. hMPV causes acute respiratory disease in children worldwide [4-6]. Currently, there are no vaccines or targeted therapies for hMPV infection. RNA interference (RNAi) is a mechanism of post-transcriptional gene silencing found in almost all eukaryotes and is triggered by endogenous small non-coding microRNAs (miRNAs) or small interfering RNAs (siRNAs) [7]. The recently development of synthetic sequence-specific siRNAs has allowed a variety of host and pathogen genes to be targeted for mRNA cleavage, effectively silencing or

\footnotetext{
* Correspondence: K.Spann@uq.edu.au

${ }^{1}$ Clinical Medical Virology Centre, The University of Queensland, Brisbane QLD 4072, Australia

${ }^{2}$ Sir Albert Sakzewski Virus Research Centre, Children's Health Services,

Brisbane QLD 4029, Australia

Full list of author information is available at the end of the article
}

significantly reducing gene expression [7]. Reports for RSV and hMPV suggest that RNAi can inhibit viral mRNA expression, and is a potentially effective therapy for respiratory infections [8-13].

siRNA molecules targeting the nucleoprotein $(\mathrm{N})$ and phosphoprotein (P) mRNA of hMPV have been found effective at inhibiting the hMPV genome [13]. Here we have designed and validated a siRNA molecule against the $\mathrm{G}$ gene, which encodes a principal attachment protein required for replication in vivo and also identified as a type I interferon (IFN) antagonist [14].

\section{Design and validation of siRNA against the $G$ gene}

The hMPV isolate CAN97-83 G gene was used as target sequence to design siRNA molecules by Dharmacon, using their algorithm and ON-TARGETplus ${ }^{\circledR}$ sense strand modification to avoid off-target effects. Two siRNA molecules were chosen for analysis and validation:

\author{
G1: sense - GCUCAAAGCAAGAGUGAAA \\ G2: sense - AGGUGAAAGUAGAGAACAUUU
}

\section{Biomed Central}

(c) 2012 Preston et al.; licensee BioMed Central Ltd. This is an Open Access article distributed under the terms of the Creative Commons Attribution License (http://creativecommons.org/licenses/by/2.0), which permits unrestricted use, distribution, and reproduction in any medium, provided the original work is properly cited. 
The molecules do not target all isolates of hMPV as identified by BLAST, due to the high level of divergence in the $\mathrm{G}$ sequence $[6,15]$. A mis-matched siRNA molecule (siMM; sense - CUAAAGUGGUAGUUGAUAUUU) was also generated as a control for the effect of transfection and off-target effects. hMPV (CAN97-83) was propagated in LLC-MK2 cells, concentrated through a 30\%/ $60 \% \mathrm{w} / \mathrm{v}$ sucrose gradient, pelleted by centrifugation at $12,000 \times g$ for 2 hours at $4{ }^{\circ} \mathrm{C}$, and the viral titre quantified using a TCID 50 assay and anti-hMPV M monoclonal antibody (Chemicon) to identify positive cells. Preliminary transfection experiments (not shown) using a fluorescein labeled RNAi delivery control (Mirus) demonstrated that transfection of $200 \mathrm{mM}$ siRNA resulted in 100\% transfection efficiency at $24 \mathrm{~h}$. Preliminary infection experiments established that $100 \%$ of cells were infected by $24 \mathrm{~h}$ post-infection (pi) when exposed to hMPV at a multiplicity of infection (MOI) of 2 in the absence of trypsin.

A549 cells were transfected with siG1, siG2 or siMM $6 \mathrm{~h}$ prior to infection with hMPV. At $12 \mathrm{~h}$ and $24 \mathrm{~h}$ pi, total RNA was extracted from cells using TRIzol/chloroform separation and RNeasy spin columns (QIAGEN). cDNA was generated using superscript III reverse transcriptase (Invitrogen) and oligo $\mathrm{d}(\mathrm{T})_{20}$ primers to select for mRNA. Quantitative (q) RT-PCR was performed to quantify $G$ mRNA reduction using the following oligonucleotides: forward primer 3' - GCAGCAATAGACATGCTCAAA-5', reverse primer 3'-GAGCTGGTGTGGTGTTCTGA-5', hybridization probe 3'-HEX-AAATCGTGTGGCACGTAGCAAATGC-BHQ- ${ }^{\prime}$. N mRNA was also quantified using the following oligonucleotides: forward primer 3' - TTACGGTGCTGGTCAAACAA-5', reverse primer 3'-TTTGGGCTTTGCCTTAAATG-5', hybridization probe 3'-HEX-CTATGACCTGGTGCGAGAAATGGGC-BHQ-5'. qPCR was performed using QuantiTECT mastermix (QIAGEN) and a Rotorgene
3000 (Roche). G and N mRNA in hMPV-infected cells was quantified in relation to $\beta$-actin mRNA for each sample and in relation to untransfected cells using the $\Delta \Delta \mathrm{Ct}$ formula. The $\Delta \Delta \mathrm{Ct}$ value was then converted to percent reduction of $\mathrm{G}$ or $\mathrm{N}$ mRNA (Applied Biosystems Technotes vol 15[2] p. 10).

At $12 \mathrm{~h} \mathrm{pi}$, siG1 and siG2 resulted in $83.7 \%$ and $85.2 \% \mathrm{G}$ mRNA reduction respectively compared to siMM (not shown). In three independent experiments, $200 \mathrm{mM}$ siG2 resulted in a mean $\mathrm{G}$ mRNA reduction of $88 \%$ (12\% remaining; $P=3.4 \times 10^{-4}$ compared to siMM) at $24 \mathrm{~h}$ pi in infected A549 cells (Figure 1a). siG1 was not as effective at reducing $\mathrm{G}$ mRNA at $24 \mathrm{~h}$ pi $(29 \%$ remaining), and was therefore not used for subsequent experiments. The effect of siG1 and siG2 on hMPV N was negligible $(<3 \%)$, indicating that other viral genes were not affected (Figure 1b).

We had planned to investigate the effect of siG2 transfection on $G$ protein expression. An antibody to detect $G$ protein in lysed cell preparations has not been described in the literature, nor is commercially available. Affinity purified rabbit antisera were generated to a $G$ peptide considered suitable for this purpose. However, the sera detected only a $\sim 32 \mathrm{kDa}$ band, which may have been a truncated form of $\mathrm{G}$, and did not detect the nontruncated $90 \mathrm{kDa}$ G protein. Due to the ambiguity of $\mathrm{G}$ protein detection we were not able to quantify the effect of $G$ mRNA reduction on G protein expression. However, other studies that involve the validation of siRNA molecules against viral mRNAs demonstrate that a reduction in target mRNA similar to that demonstrated here correlate to a significant reduction in protein $[9,10,16]$.

\section{Knockdown of $\mathrm{G}$ does not modulate viral growth in vitro or the induction of type I interferon}

Triplicate cultures of A549 cells were transfected with siG2, siMM or untransfected, as described above, and

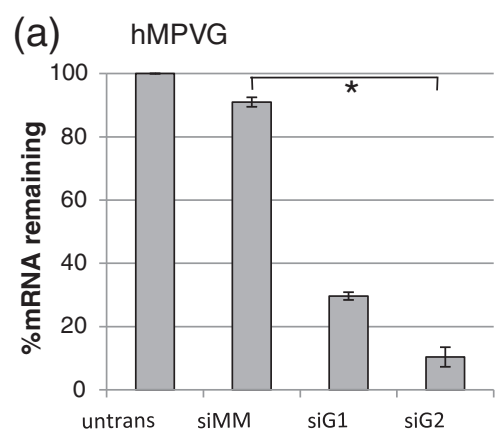

(b) hMPVN

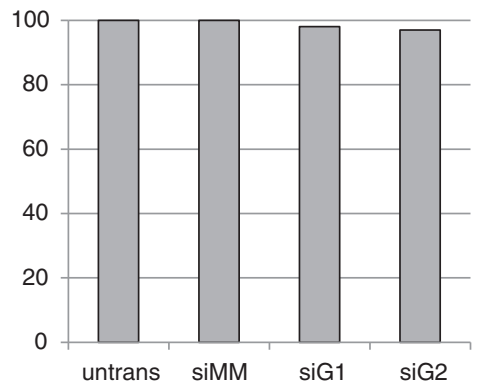

Figure 1 The effect of prior siG1 and siG2 transfection of A549 cells on G and N mRNA $24 \mathrm{~h}$ post-infection with hMPV at a MOI of 2. (a) hMPV G mRNA was significantly reduced by siG2 compared to cells either transfected with siMM ( ${ }^{*} P=1.6 \times 10^{-5}$, t-test) or not transfected. siG1 was not as effective at reducing G mRNA and subsequently was not used for experimentation. (b) siG1, siG2 and siMM did not reduce nucleoprotein (N) mRNA. hMPV N and G mRNA were quantified by RT-qPCR. Data was normalised to $\beta$-actin and expressed relative to untreated A549 cells. 


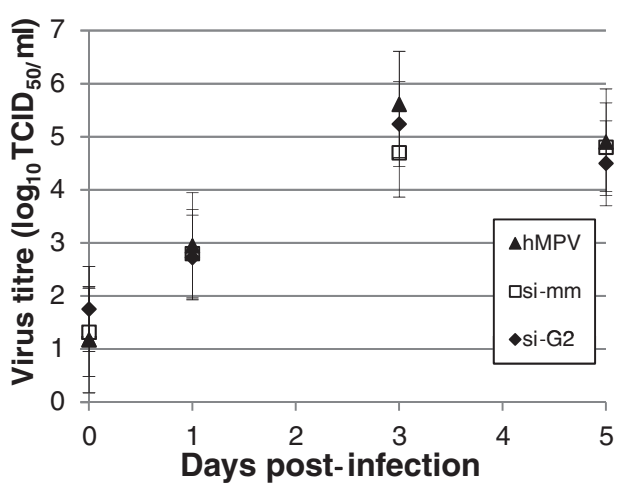

Figure 2 The effect of siG2 and siMM transfection $6 \mathrm{~h}$ prior to infection of A549 cells with hMPV at a MOI of 0.1. Viral supernatants were collected on the indicated days and the shed hMPV quantified using immunostaining and expressed as $\mathrm{TCID}_{50} / \mathrm{ml}$. A significant reduction in G mRNA did not affect hMPV shed over 5 days pi.

infected with hMPV at a MOI of 0.1. Cell supernatants were collected on days $0,1,3$ and $5 \mathrm{pi}$, and the titres of shed virus quantified by $\mathrm{TCID}_{50}$ assay. Reduction of $\mathrm{G}$ mRNA was confirmed at day 1 pi (not shown). There was no significant difference in the titres of shed virus between cells transfected with siMM, siG or untransfected, which indicated that the growth of hMPV in vitro was not affected by $\mathrm{G}$ knockdown (Figure 2; ANOVA). This correlates to a study using recombinant (r) hMPV $\triangle \mathrm{G}$ in which deletion of $\mathrm{G}$ did not attenuate viral growth in LLC-MK2 cells [17]. This differs to a report in which rhMPV $\triangle \mathrm{G}$ displayed a reduction in viral titre by one third to half a $\log$ after $24 \mathrm{~h}$ of infection in A549 cells [14].

It has been reported that deletion of $\mathrm{G}$ from rhMPV results in an increase in chemokine and type I IFN production in infected airway epithelial cells [14]. Here we investigated if knockdown of G by siRNA would also result in an increase in type I IFN induction. A549 cells were transfected with siMM or siG2, or not transfected and $6 \mathrm{~h}$ later infected at a MOI of 2 with hMPV, or mock-infected with media. UV-inactivated hMPV was used as a control for IFN induction. $24 \mathrm{~h}$ pi cells were harvested and total RNA extracted as described above. RT-qPCR was performed to quantify IFN- $\beta$ and $-\alpha$ mRNA using primers and molecular probes previously described [18]. G mRNA knockdown by $88-92 \%$ was confirmed by qPCR. The siMM and siG2 molecules alone did not induce either IFN- $\alpha$ or $-\beta$ indicating no (a)

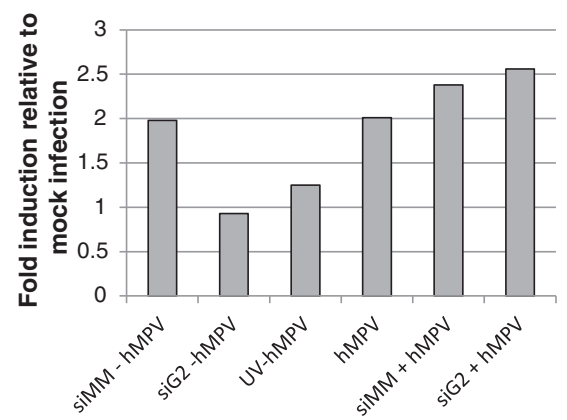

(c)

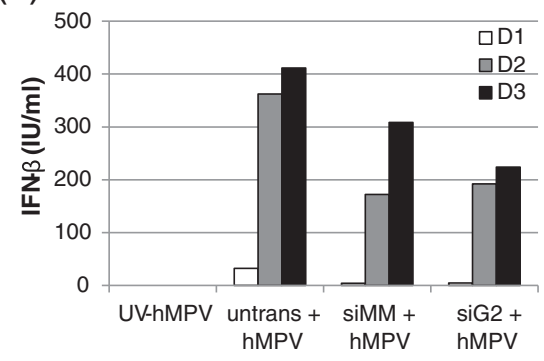

(b)

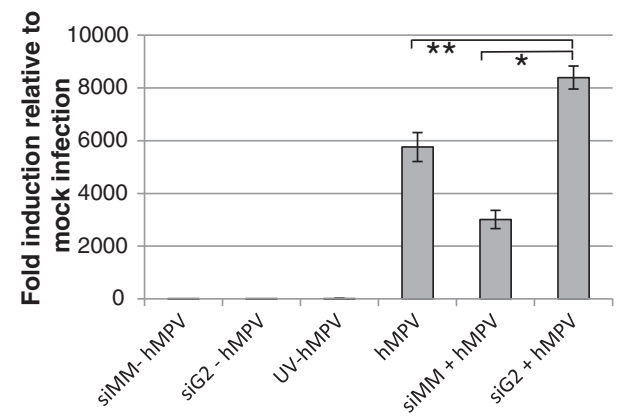

Figure 3 The effect of G knockdown on type I Interferon induction. A549 cells were transfected with siMM, siG2 or were not transfected prior to infection with hMPV at a MOI of 2, or mock infection with media. UV-inactivated hMPV was used as a control for interferon induction. Transcription of mRNA encoding human IFN-a (a) and IFN- $\beta$ (b) was measured by RT-qPCR 24 h post-infection. There was a moderately significant increase in IFN- $\beta$ mRNA in siG2 transfected cells compared to untransfected cells ( ${ }^{*} \mathrm{P}=0.03$; $\mathrm{t}$-test) and cells transfected with siMM ( ${ }^{*} \mathrm{P}=0.02$; t-test) prior to hMPV infection. Data was normalised to $\beta$-actin and expressed relative to uninfected cells. (c) A reduction in $\mathrm{G}$ did not affect IFN- $\beta$ secretion from cells transfected with either siMM or siG2 prior to hMPV infection, compared to untransfected and infected cells. Supernatants were collects on days 1,2 and 3 post-infection for ELISA analysis. 
off-target effects on type I IFN induction (Figure 3 a and $b$, first two bars). hMPV infection alone did not induce IFN- $\alpha$ and did induce IFN- $\beta$ by 5850 -fold over uninfected cells. Transfection with siMM prior to hMPV infection (siMM + hMPV) dampened IFN- $\beta$ induction indicating some effect of transfection on the cells. However, the knockdown of $G$ resulted in a marginally significant increase in IFN- $\beta$ mRNA induction compared to hMPV infection alone $(P=0.03)$. A more significant increase was observed compared to siMM +hMPV $(P=0.02)$, although the difference in fold induction between these two treatments, from 3000-fold (siMM) to 8500 -fold (siG2), was only 2.85 , which may be of only marginal biological significance. ELISA was performed on the cell supernatants collected from transfected and/ or infected cells at $24 \mathrm{~h}, 48 \mathrm{~h}$ and $72 \mathrm{~h}$ pi. A reduction of $G$ mRNA did not result in a significant increase in secreted IFN- $\beta$ at any time (Figure 3c; $P>0.05$ ). In fact, a slight reduction in secreted IFN- $\beta$ is observed, which may be the result of transfection. This suggests that although there is a moderate increase in IFN $-\beta$ mRNA when $G$ mRNA is reduced, this does not result in an increase in IFN- $\beta$ protein secreted from infected cells. This observation differs from another study in which there was a significant increase in IFN- $\alpha / \beta$ secretion from infected cells when $G$ was deleted from rhMPV. ELISA (not shown) for IFN- $\alpha$ was conducted and showed no detectable induction as correlated to qPCR results (Figure 3a). Our growth curves for hMPV are similar to those of Biacchesi et al., (2004), which also suggest that a knockdown of $\mathrm{G}$ does not induce type I IFN, which may attenuate viral growth.

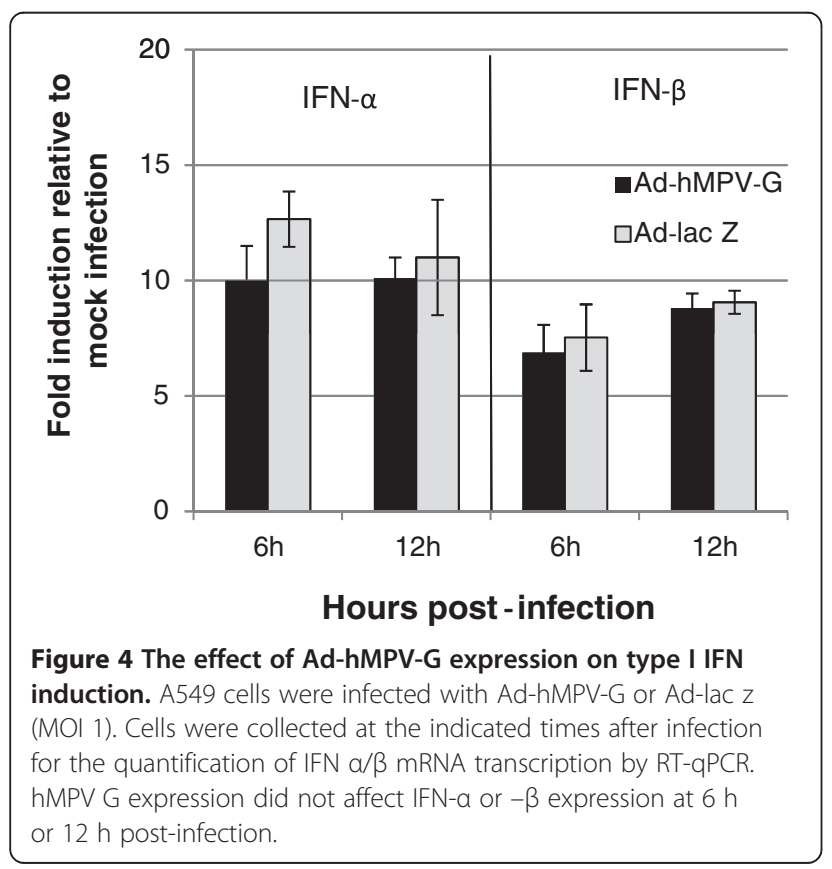

The effect of $G$ on type I IFN induction was also investigated using an adenovirus which expressed the hMPV G protein (Ad-hMPV-G; gift from R. Tripp, University of Georgia, Athens, USA). A549 cells were infected with Ad-hMPV G or Ad-Lac Z as a control, at a MOI of 1 . After $1 \mathrm{~h}$ adsorbtion at $37{ }^{\circ} \mathrm{C}$, virus inoculum was replaced with growth media and infected cells collected for mRNA analysis at $6 \mathrm{~h}$ and $12 \mathrm{~h}$ pi. G protein expression by adenovirus did not significantly affect type I IFN mRNA levels compared to control (Figure 4).

In summary, we have designed and validate a siRNA molecule that is effective against the G gene of hMPV in vitro. A significant reduction in G mRNA did not reduce viral growth in vitro or induce a significant type I IFN response, suggesting that G may not be a significant type I IFN antagonist, and other hMPV proteins may play a role in modulating type I IFN induction. However hMPV G may still be a valid target for RNAi as G is required for viral replication in vivo [17].

\section{Competing interests}

The authors declare that they have no competing interests.

\section{Acknowledgements}

This work was funded by the Australian National Health and Medical Research Council, grant number 508601.

\section{Author details}

${ }^{1}$ Clinical Medical Virology Centre, The University of Queensland, Brisbane QLD 4072, Australia. 'Sir Albert Sakzewski Virus Research Centre, Children's Health Services, Brisbane QLD 4029, Australia. ${ }^{3}$ Faculty of Applied Science, University of Canberra, Canberra ACT 0200, Australia. ${ }^{4}$ Institute for Glycomics, Griffith University, Gold Coast QLD 4222, Australia.

\section{Authors' contributions}

KMS and SM conceived of the study. FMP, CPS and RR performed the experiments. FMP and KMS wrote the manuscript. All authors read and approved the final manuscript.

Received: 3 November 2011 Accepted: 7 June 2012

Published: 7 June 2012

\section{References}

1. van den Hoogen BG, de Jong JC, Groen J, Kuiken T, de Groot R, Fouchier RA, Osterhaus AD: A newly discovered human pneumovirus isolated from young children with respiratory tract disease. Nat Med 2001, 7:719-724.

2. Principi N, Bosis S, Esposito S: Human metapneumovirus in paediatric patients. Clin Microbiol Infect 2006, 12:301-308.

3. Williams JV, Harris PA, Tollefson SJ, Halburnt-Rush LL, Pingsterhaus JM, Edwards KM, Wright PF, Crowe JE Jr: Human metapneumovirus and lower respiratory tract disease in otherwise healthy infants and children. $N$ Engl J Med 2004, 350:443-450.

4. Biovin G, Abed Y, Pelletier G, Ruel L, Moisan D, Cote S, Peret TC, Erdman $D D$, Anderson L: Virological features and clinical manifestations associated with human metapneumovirus: a new paramyxovirus responsible for acute respiratory-tract infections in all age groups. $J$ Infect Dis 2002, 186:1330-1334.

5. Greensill J, McNamara PS, Dove W, Flanagan B, Smyth RL, Hart CA: Human metapneumovirus in severe respiratory syncytial virus bronchiolitis. Emerg Infect Dis 2003, 9:372-375.

6. van den Hoogen BG, Herfst S, Sprong L, Cane PA, Forleo-Neto E, de Swart $\mathrm{RL}$, Osterhaus AD, Fouchier RA: Antigenic and genetic variability of human pneumoviruses. Emerg Infect Dis 2004, 10:658-666. 
7. Barik S: Control of nonsegmented negative-strand RNA virus replication by siRNA. Virus Research 2004, 102:27-35.

8. Barik $S$, Bitko V: Prospects of RNA interference therapy in respiratory viral diseases: update 2006. Expert Opin Biol Ther 2006, 6:1151-1160.

9. Bitko V, Barik S: Phenotypic silencing of cytoplasmid genes using sequence-specific double-stranded short interfering RNA and its application in the reverse genetics of wild type negative-strand RNA viruses. BMC Microbiology 2001, 1:34.

10. Bitko V, Musiyenko A, Shulyayeva O, Barik S: Inhibition of respiratory viruses by nasally administered siRNA. Nature Medicine 2005, 11(1):50-55.

11. Zhang W, Yang H, Knog X, Mahapatra S, San Juan-Vergara H, Hellermann G, Behera S, Singam R, Lockey RF, Mahapatra SS: Inhibition of respiratory syncytial virus infection with intranasal siRNA nanoparticles targeting the viral NS1 gene. Nature Medicine 2005, 11(1):56-62.

12. Kong X, Zhang W, Lockey RF, Auais A, Piedimonte G, Mohapatra: Respiratory syncytial virus infection in Fisher 344 rats is attenuated by short interfering RNA against the RSV-NS1 gene. Genetic Vaccines and Therapy 2007, 5:4.

13. Deffrasnes C, Cavahagh MH, Goyette N, Cui K, Ge Q, Seth S, Templin MV, Quay SC, Johnson PH, Boivin G: Inhibition of human metapneumovirus replication by small interfering RNA. Antiviral therapy 2008, 13:821-832.

14. Bao X, Liu T, Shan Y, Li K, Garofalo RP, Casola A: Human metapneumovirus glycoprotein G inhibits innate immune responses. PLoS Pathog 2008, 4(5).

15. Peret TC, Abed Y, Anderson $\sqcup$, Erdman DD, Boivin G: Sequence polymorphism of the predicted human metapneumovirus $G$ glycoprotein. J Gen Virol 2004, 85:679-686.

16. Munir S, Kaur K, Kapur V: Avian metapneumovirus phosphoprotein targeted RNA interference silences the expression of viral proteins and inhibits virus replication. Antivir Res 2006, 69:46-51.

17. Biacchesi S, Skiadopoulos MH, Yang L, Lamirande EW, Tran KC, Murphy BR, Collins PL, Buchholz UJ: Recombinant human metapneumovirus lacking the small hydrophobic $\mathrm{SH}$ and/or attachment $\mathrm{G}$ glycoprotein: deletion of $\mathrm{G}$ yields a promising vaccine candidate. J Virol 2004, 78:12877-12887.

18. Spann KM, Tran KC, Chi B, Rabin RL, Collins PL: Suppression of the induction of alpha, beta and lambda interferons by the NS1 and NS2 proteins of human respiratory syncytial virus in human epithelial cells and macrophages. J Virol 2004, 78:4363-4369.

doi:10.1186/1743-422X-9-105

Cite this article as: Preston et al:: siRNA against the $\mathrm{G}$ gene of human metapneumovirus. Virology Journal 2012 9:105.

\section{Submit your next manuscript to BioMed Central and take full advantage of:}

- Convenient online submission

- Thorough peer review

- No space constraints or color figure charges

- Immediate publication on acceptance

- Inclusion in PubMed, CAS, Scopus and Google Scholar

- Research which is freely available for redistribution 